Purdue University Purdue e-Pubs

6-13-2006

\title{
Hydrodynamic Loading of Microcantilevers Vibrating in Viscous Fluids
}

Sudipta Basak

Arvind Raman

S V. Garimella

Purdue University, sureshg@purdue.edu

Follow this and additional works at: http:/ / docs.lib.purdue.edu/coolingpubs

Basak, Sudipta; Raman, Arvind; and Garimella, S V., "Hydrodynamic Loading of Microcantilevers Vibrating in Viscous Fluids" (2006). CTRC Research Publications. Paper 51.

http://dx.doi.org/10.1016/j.ijmultiphaseflow.2006.03.002

This document has been made available through Purdue e-Pubs, a service of the Purdue University Libraries. Please contact epubs@purdue.edu for additional information. 


\title{
Hydrodynamic loading of microcantilevers vibrating in viscous fluids
}

\author{
Sudipta Basak and Arvind Raman ${ }^{\text {a) }}$ \\ Birck Nanotechnology Center and School of Mechanical Engineering, Purdue University, \\ West Lafayette, Indiana 47907-2088 \\ Suresh V. Garimella \\ School of Mechanical Engineering, Purdue University, West Lafayette, Indiana 47907-2088
}

(Received 27 October 2005; accepted 6 April 2006; published online 13 June 2006)

\begin{abstract}
The hydrodynamic loading of elastic microcantilevers vibrating in viscous fluids is analyzed computationally using a three-dimensional, finite element fluid-structure interaction model. The quality factors and added mass coefficients of several modes are computed accurately from the transient oscillations of the microcantilever in the fluid. The effects of microcantilever geometry, operation in higher bending modes, and orientation and proximity to a surface are analyzed in detail. The results indicate that in an infinite medium, microcantilever damping arises from localized fluid shear near the edges of the microcantilever. Closer to the surface, however, the damping arises due to a combination of squeeze film effects and viscous shear near the edges. The dependence of these mechanisms on microcantilever geometry and orientation in the proximity of a surface are discussed. The results provide a comprehensive understanding of the hydrodynamic loading of microcantilevers in viscous fluids and are expected to be of immediate interest in atomic force microscopy and microcantilever biosensors. (C) 2006 American Institute of Physics.
\end{abstract}

[DOI: $10.1063 / 1.2202232]$

\section{INTRODUCTION}

Understanding the hydrodynamic loading on microcantilevers vibrating in viscous fluids is crucial for the improved performance of atomic force microscopy (AFM) in liquids and of a variety of microelectro mechanical systems (MEMS) including biosensors and actuators. For instance, the AFM based imaging, manipulation, and property characterization of biological molecules in liquids are a rapidly growing field. ${ }^{1-4}$ The imaging resolution and force exerted on the sample are directly correlated to the quality factor of the AFM microcantilever in the surrounding liquid. ${ }^{5}$ Any improvement in the microcantilever quality factor through mechanical design would be a significant advance in this area. Similarly, in the area of microcantilever-based biosensing ${ }^{6-8}$ the binding of target analyte molecules to the microcantilever is often detected via a shift in the microcantilever resonance frequency induced due to added molecular mass. For such biosensors the minimum detectable molecular mass is inversely proportional to the quality factor. ${ }^{9,10}$ The detection of the binding of single biological molecules in liquid environments is arguably the grand goal of microcantilever biosensors and could impact significantly the field of genomics and proteomics. 7,11 This goal is severely challenged by the low quality factors and the low frequencies of microcantilevers in liquids. Clearly, the design of microcantilevers to maximize quality factors and resonance frequencies in liquids is of significant interest in the AFM and biosensing communities.

Broadly speaking, the presence of a surrounding fluid influences significantly the linear vibration characteristics of

\footnotetext{
a) Author to whom correspondence should be addressed; electronic mail: raman@ecn.purdue.edu
}

the microcantilever such as the damping and natural frequencies of different modes. The dissipation of the vibration energy of a microcantilever can occur due to acoustic radiation damping, internal structural damping, viscous losses in the surrounding fluid, and support loss. ${ }^{12}$ However, at the micrometer scale, viscous losses in the surrounding fluid are typically two to three orders of magnitude greater than other losses combined. ${ }^{13,14}$ The surrounding fluid also affects the natural frequencies of the microcantilever. This effect is quantified by the so called added-mass coefficient of the surrounding fluid on the microcantilever. $\mathrm{Chu}^{15}$ derived approximate expressions for the ratio of wet (in fluid) and dry (in vacuo) frequencies of the bending and torsional modes of cantilevers with rectangular cross sections submerged in an inviscid fluid. However, Chu's ${ }^{15}$ analysis is inapplicable at small scales where the fluid viscosity increasingly affects its motion around the vibrating microcantilever. ${ }^{16-18}$

Several authors have attempted to predict analytically and semianalytically the quality factors and wet (in fluid) natural frequencies of microcantilevers in viscous fluids. In Refs. 14 and 19-21, the microcantilever was modeled as a sphere or a string of spheres. However, these models are inaccurate for high density fluids such as water or acetone. In Refs. 16 and 18 a semianalytical theory was developed to predict the quality factor of bending and torsional vibrations of microcantilevers vibrating in a viscous fluid of infinite extent. However, it was assumed that the surrounding fluid flows two dimensionally in the cross-sectional plane of the microcantilever. This restricts the semianalytical solution to only the lowest modes of vibration because it neglects fluid flow along the axis of the microcantilever. In fact, recent experimental work by Maali et al. ${ }^{22}$ has shown that the errors 
in estimation of the damping and added mass coefficients using the semianalytical model ${ }^{16,18}$ increase with increase in mode number.

Finally, when the microcantilever is brought close to a surface, the squeeze film effect between the microcantilever and the surface contributes significantly to the damping, ${ }^{12}$ in addition to the fluid dissipation at the edges and above the microcantilever. Recently Green and Sader ${ }^{23}$ derived an elegant semianalytical model to predict the hydrodynamic functions ${ }^{24}$ for microcantilevers vibrating close to a surface. However, their method is based on a similar formulation as in Ref. 16 and thus suffers from the same limitations. Moreover the quality factors are not computed explicitly and the model is not applicable for microcantilevers inclined to a surface-a situation commonly found in AFM applications.

This paper develops a fully three-dimensional finite element flow-structure interaction model to predict the hydrodynamic loading of microcantilevers in fluids. A threedimensional linearly elastic microcantilever interacts with the surrounding fluid which is modeled using the threedimensional, incompressible Navier-Stokes equations. This approach overcomes the limitations of the conventional analytical and semianalytical models and demonstrates clearly the physics of dissipation and added mass. The main energy dissipation mechanisms and their locations on the fluid structure interaction boundary are studied. The effects of microcantilever geometry, orientation, higher mode operation, and the presence of a nearby surface are investigated in detail. The results are comprehensive and are expected to aid in the ongoing design of microcantilevers for advanced AFM and biosensing applications.

\section{THE COMPUTATIONAL MODEL}

All the numerical computations in this paper are performed using fluid structure interaction (FSI) codes in ADINA. $^{25}$ The microcantilever is submerged in a threedimensional enclosure of Navier-Stokes elements of an incompressible viscous fluid. These Navier-Stokes fluid elements are then surrounded by potential-based threedimensional fluid elements which terminate in an infinite potential wall. For the case of microcantilevers submerged in a fluid of infinite extent, the model is shown schematically in Fig. 1(a). Figure 1(b) is the two-dimensional schematic when the microcantilever vibrates close to a surface and its threedimensional cut image is shown in Fig. 1(c). Figure 1(d) is a two-dimensional schematic when the microcantilever vibrates inclined to the nearby surface.

The FSI ADINA model is developed in three different domains: (i) the solid domain, (ii) the viscous fluid domain, and (iii) the inviscid potential flow domain. In the solid domain, the model consists of three-dimensional elastic elements. Surrounding the solid domain is the viscous fluid domain which is modeled using three-dimensional viscous NavierStokes elements. The viscous fluid domain is in turn either (a) entirely bounded by the inviscid potential flow domain in the case of a microcantilever in a fluid of infinite extent, or (b) bounded by the inviscid potential flow domain and a rigid surface for the case when the microcantilever is in close

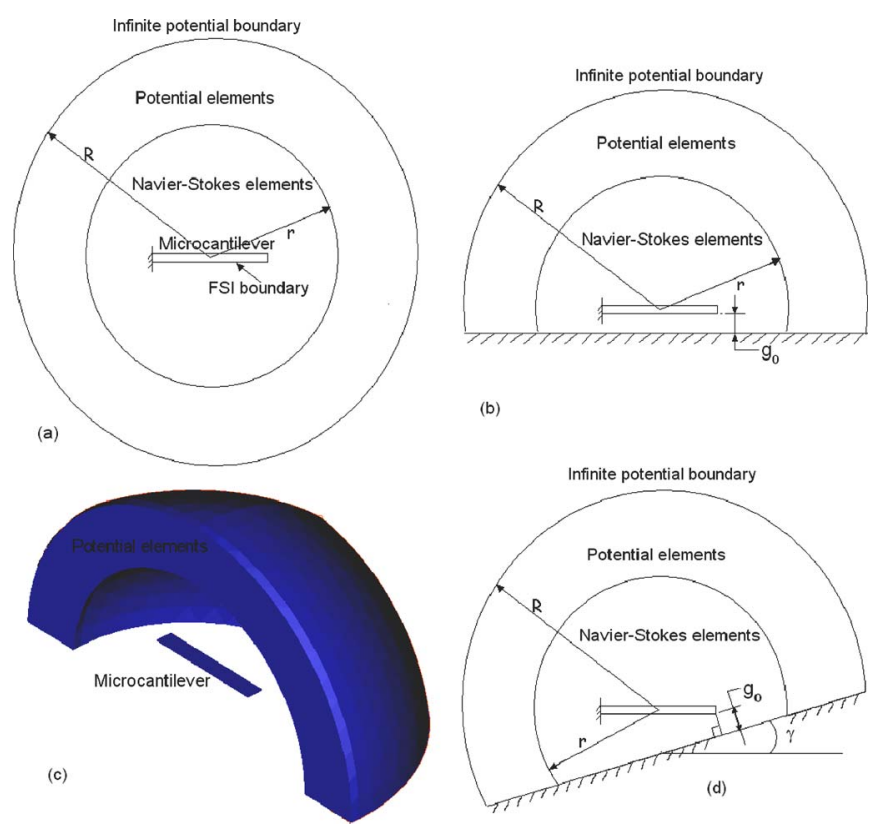

FIG. 1. (Color online) (a) A cross-sectional schematic of the threedimensional finite element model when the microcantilever is far from any surface. (b) A cross-sectional schematic of the finite element model when a microcantilever vibrates parallel to a surface. (c) A three-dimensional cut image of the finite element model in ADINA when the microcantilever is close to a surface. (d) A cross-sectional schematic of the finite element model when the microcantilever is inclined close to a surface.

proximity to a surface. Finally the inviscid potential domain is terminated at an infinite potential boundary. Two fluidstructure boundaries need to be defined for this model; one between the microcantilever and the Navier-Stokes fluid elements and another between the Navier-Stokes elements and the potential-based fluid elements. Fluid velocities are restricted to be normal to the rigid wall at the interface between the potential elements and the rigid wall, whereas noslip boundary conditions are enforced at the rigid wall and the viscous fluid interface as well as the interface between the microcantilever and the viscous fluid. The microcantilever is clamped at its left end.

\section{A. Convergence study}

A thorough convergence study is performed to verify domain independence [dependence on $r$ and $R$, in Fig. 1(a)], mesh independence, and time-step independence of the predicted results. The results of this convergence study can be found in Appendix A. Based on this detailed convergence study, all the numerical computations for the three-dimensional model performed in this paper use $r=0.9 \sqrt{3 / 2} a$ and $R=1.5 \sqrt{3 / 2} a$ with a step between $T / 50$ and $T / 40$, and with nearly 105000 elements in the viscous fluid domain when the microcantilever vibrates in a fluid of infinite extent.

\section{B. Nondimensional terms and benchmarking}

The predictions of the computational model developed in the paper are compared with previously published results $^{17,18,26}$ in Appendix B. Here we record certain nondimensional quantities that are needed for making a fair com- 
parison. The fluid flow around the microcantilever is governed by the incompressible Navier-Stokes equations,

$$
\rho \frac{\partial \mathbf{v}}{\partial t}+\rho(\mathbf{v} \cdot \nabla) \mathbf{v}=-\nabla p+\mu \nabla^{2} \mathbf{v},
$$

where $\rho, \mathbf{v}, p$, and $\mu$ are the density, velocity, pressure, and viscosity of the fluid, respectively. If $b$ and $\omega$ represent the width and damped natural frequency of the microcantilever in the surrounding fluid, then the nondimensional term formed by dividing the temporal derivative term in Eq. (1) by the viscous term yields the modified Reynolds number ${ }^{27}$ (referred to as Reynolds number in Ref. 16 and as nondimensional frequency $\beta$ in Ref. 24).

$$
\operatorname{Re}=\frac{|\rho \partial \mathbf{v} / \partial t|}{4 \mid \mu \nabla^{2} \mathbf{v}}=\frac{\rho \omega b^{2}}{4 \mu} .
$$

Here the spatial gradient of velocity along the width of the microcantilever is assumed to dominate the overall velocity gradient. The factor of 4 is introduced to be consistent with the definition of the nondimensional term $\beta$ of Ref. 24. Another useful nondimensional term from Ref. 16 is

$$
\Pi=\frac{\rho b}{\rho_{b} h} .
$$

$\Pi$ can be regarded as the ratio of the added mass of the fluid to the mass of the microcantilever.

In comparison to the previously published literature $^{17,18,26}$ (see Appendix B), the ADINA predicted quality factors and frequencies are within $10 \%$ of those found in experiments for a wide range of cantilevers in air, water, carbon-tetrachloride $\left(\mathrm{CCl}_{4}\right)$ and acetone.

\section{MICROCANTILEVER VIBRATING IN A VISCOUS FLUID OF INFINITE EXTENT}

Having benchmarked the ADINA model predictions against prior experimental and theoretical results, we now turn our attention to understanding the fundamental mechanisms of hydrodynamic loading on microcantilevers. Specifically, we investigate the effects of complicated microcantilever geometry such as slots or nonuniformity in the microcantilever width on the quality factors and added mass coefficients. The most important parameter in the twodimensional semianalytical model $1^{16,18,24}$ is the dominant length scale, based on which the modified Reynolds number is calculated. This dominant length scale over which fluid shear occurs is easily identified as the width for uniform, rectangular microcantilevers. However, in the case of more complicated geometries, the dominant length scale is less straightforward to identify. Consequently the semianalytical approaches of Refs. 16 and 26 cannot easily be applied to the complicated microcantilever geometries considered here. We will show that not only does the computational model offer a better understanding of the fundamental sources of hydrodynamic loading, but it can also help identify the critical length scales which enable the use of the semianalytical models for more complicated geometries.

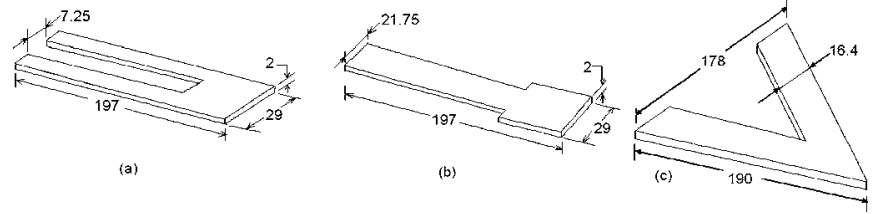

FIG. 2. (Color online) Schematics of (a) the slotted microcantilever C3 with slot width equal to $25 \%$ of the overall microcantilever width, (b) of the paddle microcantilever $\mathrm{C} 4$ with neck width equal to the width of $25 \%$ of the overall microcantilever width, and (c) of the triangular microcantilever C5 of nominal dimensions. All dimensions are in micrometer.

\section{A. Simplified formula for microcantilevers vibrating in free fluid}

Prior to studying the effect microcantilever geometry on hydrodynamic loading using our finite element model, we briefly review an approximation of the semianalytical theory of Ref. 16. The hydrodynamic function, $\Gamma(j \omega),{ }^{28}$ representing the real and imaginary parts of the pressure of the surrounding on an oscillating rectangle in two dimensions, can be approximated for the bending modes as ${ }^{22}$

$$
\begin{aligned}
& \Gamma=\Gamma_{r}+j \Gamma_{i}, \\
& \Gamma_{r}=a_{1}+a_{2} \frac{\delta}{b}=a_{1}+\frac{a_{2}}{\sqrt{\operatorname{Re}}}, \\
& \quad \Gamma_{i}=b_{1} \frac{\delta}{b}+b_{2}\left(\frac{\delta}{b}\right)^{2}=\frac{b_{1}}{\sqrt{\operatorname{Re}}}+\frac{b_{2}}{\operatorname{Re}},
\end{aligned}
$$

where $a_{1}=1.0553, a_{2}=3.7997, b_{1}=3.8018, b_{2}=2.7364$, and $\delta=\sqrt{2 \mu / \rho \omega_{\text {wet }}}$ is the thickness of the unsteady boundary layer on the microcantilever surface. Wet natural frequencies and quality factors for bending modes can be approximated as $^{16}$

$$
Q_{b}^{\infty}=\frac{\left(4 \rho_{b} h / \pi \rho b\right)+\Gamma_{r}\left(\omega_{\mathrm{wet}}\right)}{\Gamma_{i}\left(\omega_{\mathrm{wet}}\right)}=\frac{(4 / \pi)(1 / \Pi)+\Gamma_{r}\left(\omega_{\mathrm{wet}}\right)}{\Gamma_{i}\left(\omega_{\mathrm{wet}}\right)},
$$

$$
\begin{aligned}
\omega_{\text {wet }, b} & =\frac{\omega_{\mathrm{dry}}}{\sqrt{1+(\pi / 4)\left(\rho b / \rho_{b} h\right) \Gamma_{r}\left(\omega_{\mathrm{wet}}\right)}} \\
& =\frac{\omega_{\mathrm{dry}}}{\sqrt{1+(\pi / 4) \Pi \Gamma_{r}\left(\omega_{\mathrm{wet}}\right)}},
\end{aligned}
$$

where $\omega_{\text {dry }}$, the natural frequency in vacuum, can be determined analytically for each mode of the microcantilever. The added mass coefficients are computed as

$$
\alpha_{m}=\left(\frac{\omega_{\mathrm{dry}}}{\omega_{\mathrm{wet}}}\right)^{2}-1
$$

In what follows we will use this approximate form for the semianalytical model to compare it against the finite element model.

\section{B. Quality factor and its dependence on microcantilever geometry}

While the existing semianalytical models ${ }^{16,18}$ provide useful tools to predict the quality factors and added mass 
TABLE I. Effect of geometry on quality factors and added mass coefficients of microcantilevers vibrating in water. The predicted quality factors, wet, and dry natural frequencies for the first bending mode for the cantilevers in Fig. 2(a) (C3), Fig. 2(b) (C4), and Fig. 2(c) are shown below.

\begin{tabular}{|c|c|c|c|c|c|c|c|c|c|c|}
\hline \multirow[b]{2}{*}{$\begin{array}{l}\text { Microcantilever } \\
\text { slot/neck width }\end{array}$} & \multicolumn{3}{|c|}{$\mathrm{C} 3$} & \multicolumn{3}{|c|}{$\mathrm{C} 4$} & \multicolumn{4}{|c|}{$\mathrm{C} 5$} \\
\hline & $Q$ & $\begin{array}{c}f^{\text {wet }} \\
(\mathrm{kHz})\end{array}$ & $\begin{array}{c}f^{\mathrm{dry}} \\
(\mathrm{kHz})\end{array}$ & $Q$ & $\begin{array}{c}f^{\text {wet }} \\
(\mathrm{kHz})\end{array}$ & $\begin{array}{c}f^{\mathrm{dry}} \\
(\mathrm{kHz})\end{array}$ & $\begin{array}{l}\text { Arm width } \\
\quad(\mu \mathrm{m})\end{array}$ & $Q$ & $\begin{array}{c}f^{\text {wet }} \\
(\mathrm{kHz})\end{array}$ & $\begin{array}{l}f^{\text {dry }} \\
\text { (dry) }\end{array}$ \\
\hline No slot/neck & 3.7 & 24.6 & 70.9 & 3.7 & 24.6 & 70.9 & $\cdots$ & $\ldots$ & $\cdots$ & $\cdots$ \\
\hline $25 \%$ & 2.9 & 22.9 & 64.7 & 3.5 & 22.3 & 64.7 & 8.2 & 0.9 & 4.6 & 29.0 \\
\hline $50 \%$ & 2.7 & 20.5 & 56.0 & 3.3 & 20.0 & 57.3 & 16.4 & 1.3 & 5.0 & 24.6 \\
\hline $75 \%$ & 2.6 & 15.2 & 42.3 & 2.7 & 15.1 & 42.3 & 30 & 1.9 & 5.3 & 22.2 \\
\hline
\end{tabular}

coefficients of microcantilevers in liquids of infinite extent, the fundamental mechanisms of fluid dissipation and their locations remain poorly understood. In order to understand these fundamental mechanisms, we explore the hydrodynamic loading of two different geometries of microcantilevers vibrating in water. The three types of geometry considered are shown in Fig. 2: the slotted microcantilever (henceforth referred to as $\mathrm{C} 3$ ), of Fig. 2(a), the paddle shaped microcantilever (henceforth referred to as $\mathrm{C} 4$ ), of Fig. 2(b), and the triangular microcantilever of Fig. 1(c) (henceforth referred to as $\mathrm{C} 5$ ). Both the $\mathrm{C} 3$ and $\mathrm{C} 4$ microcantilevers can be thought of as being derived from the same uniform rectangular microcantilever $\mathrm{C} 2$; for $\mathrm{C} 3$, a slot of given width is made in the otherwise uniform microcantilever, while in $\mathrm{C} 4$, material is removed from the microcantilever edges to create a neck for the paddle. The geometric and material properties of the triangular microcantilever are taken from Ref. 29. This microcantilever is made of silicon nitride $(\rho=2800 \mathrm{~kg} / m 3, E=130 \mathrm{GPa}$, and $\nu=0.25)$ and has nominal dimensions $w=16.4 \mu \mathrm{m}, \quad t=0.64 \mu \mathrm{m}, \quad b=178 \mu \mathrm{m}$, and $L=190 \mu \mathrm{m}$. In what follows we keep all other dimensions constant and vary the width of the C5 microcantilever.

Let us first consider the variation of the quality factors of the $\mathrm{C} 3$ and $\mathrm{C} 4$ microcantilevers in the first bending mode in water, which are presented for increasing the slot width in $\mathrm{C} 3$ and decreasing the neck width in C4 in Table I. Two important observations may be made from the table. First, the quality factor decreases with increasing slot width in $\mathrm{C} 3$ or decreasing neck width in $\mathrm{C} 4$ compared to the unmodified microcantilever C2. Second, for the same surface area, the quality factors for the $\mathrm{C} 4$ type microcantilevers are greater than for the $\mathrm{C} 3$ type. These are nonintuitive results which we will now explore by analyzing the fundamental dissipation mechanisms of these microcantilevers in a fluid.

To explain these results, the shear stress profiles in the fluid are plotted on specific cross-sectional planes as shown in Fig. 3. These shear stress profiles are computed as part of the microcantilever transient motion used for determining the quality factors. Specifically, the shear stress distribution in the surrounding fluid on specific cross-sectional planes is computed at a time instant following the first cycle of oscillation when the microcantilever is at its undeflected position but in the process of moving upwards during its transient motion. From Fig. 3 it is clear that the greatest shear stresses in the fluid are found near all the edges of the microcantilevers. Moreover, because the total edge length of the slotted microcantilever $\mathrm{C} 3$ is greater compared to the unmodified $\mathrm{C} 2$ microcantilever, the quality factor is lesser for the $\mathrm{C} 3$ microcantilever in comparison to the unmodified $\mathrm{C} 2$ microcantilever.

The computational results for the $\mathrm{C} 3$ and $\mathrm{C} 4$ microcantilevers can be further analyzed as follows. For the solid rectangular microcantilever $\mathrm{C} 2$ shown in Figs. 3(a) and 3(b), it is clear that the largest velocity gradient in the fluid is dictated by the shortest dimension over which the fluid shear changes the most. Therefore, for a solid rectangular microcantilever, the appropriate length scale to use in the semianalytical model ${ }^{16}$ is its width. By contrast, for the $\mathrm{C} 3$ microcantilever shown in Figs. 3(c) and 3(d), the fluid shear localizes at all edges. Consequently, the appropriate dimension to use in the semianalytical model would be the width of each arm of the slotted $\mathrm{C} 3$ microcantilever and the neck width of the $\mathrm{C} 4$ paddle microcantilever. It follows then that the effective width of $\mathrm{C} 3$ and $\mathrm{C} 4$ microcantilevers is lesser than that of the unmodified microcantilever $\mathrm{C} 2$. In a similar manner, for both the $\mathrm{C} 3$ and $\mathrm{C} 4$ microcantilevers fluid shear stresses develop over smaller widths thereby lowering the effective width of these microcantilevers. Microcantilever $\mathrm{C} 3$, on the other hand, has a smaller effective width compared to the $\mathrm{C} 4$ microcantilever because high shear stresses

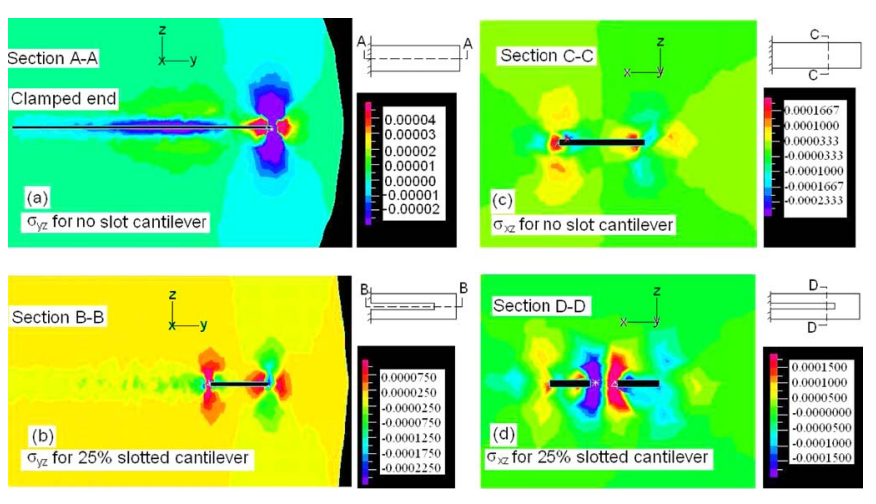

FIG. 3. (Color online) (a) Shear stress $\left(\tau_{y z}\right)$ in the surrounding liquid (water) in the $y z$ plane for $\mathrm{C} 2$ (unslotted) microcantilever along the centerline of the microcantilever, (b) shear stress $\left(\tau_{y z}\right)$ in the $y z$ plane for $25 \%$ slotted microcantilever along the centerline of the microcantilever, (c) shear stress $\left(\tau_{x z}\right)$ in the $x z$ plane for C2 microcantilever in the lateral cross section of $140 \mu \mathrm{m}$ from the clamp, and (d) shear stress $\left(\tau_{x z}\right)$ in the $x z$ plane for the $25 \%$ slotted microcantilever of $140 \mu \mathrm{m}$ from the clamp. All stress profiles in this figure are for the first bending mode in water. The microcantilever axis is oriented along the $y$ direction. These stress profiles are plotted at the instant the microcantilever is deforming upwards after one complete cycle of oscillation. Shear stresses are in Pascals. 
also develop at the inner edges of the $\mathrm{C} 3$ microcantilever in addition to the outer edges. Also, it can be shown using the semianalytical model ${ }^{16}$ and from Eqs. (2), (4), and (5a) that a decrease in effective width leads to a decrease in quality factor. This is because the ratio of the real and imaginary parts of the hydrodynamic function $\Gamma(j \omega)[\mathrm{Eq} .(4)]$ increases with an increase in $b / \delta$. Because $\delta$ is independent of the microcantilever width, the quality factor increases with increasing width. Finally because the effective width of the $\mathrm{C} 4$ microcantilever is greater than that for the $\mathrm{C} 3$ microcantilever, its quality factors are also greater compared to the $\mathrm{C} 3$ microcantilever. In this manner, the computational model can be used to identify crucial geometrical dimensions to be used in the semianalytical model, especially when the microcantilever possesses a complex geometry. The results for the triangular C5 microcantilever also follow a similar trend. The quality factors of the first bending mode decrease with decreasing arm width (Table I).

\section{Added mass coefficients and their dependence on microcantilever geometry}

In addition to viscous dissipation, the surrounding fluid also creates an added mass effect on the microcantilever. This results in wet natural frequencies that are much lesser than the dry natural frequencies and limits the efficiency of those microcantilevers for applications which depend on the frequency of operation. The dry frequencies and the wet natural frequencies in water are calculated for the $\mathrm{C} 3$ and the C4 microcantilevers for different slot and neck widths and listed in Table I. The wet natural frequencies of both the C3 and $\mathrm{C} 4$ microcantilevers are similar in value they possess similar surface areas. The wet natural frequencies follow the same trend with increasing slot widths or decreasing neck widths. Specifically, the dry frequencies decrease with increasing slot width or decreasing neck width because removing material from the microcantilever $\mathrm{C} 2$ to form $\mathrm{C} 3$ and $\mathrm{C} 4$ results in a greater reduction in stiffness of the microcantilever in comparison to the reduction in modal mass. Thus there is only a slight difference in fluid added mass effects between the $\mathrm{C} 3$ and $\mathrm{C} 4$ microcantilevers. For the $\mathrm{C} 5$ triangular microcantilevers [Fig. 2(c)], the wet natural frequencies decrease with decreasing arm width following the same trend as the $\mathrm{C} 3$ and $\mathrm{C} 4$ microcantilevers.

\section{Designing microcantilevers in fluid environment for optimal performance}

We now discuss the use of the computational model developed in this work in the practical design of microcantilevers operating in liquid or gaseous environments. In both microcantilever biosensing and liquid AFM applications, the general goal is to (a) increase the quality factor and (b) increase the wet natural frequency when immersed in a fluid. These may be supplemented by additional requirements to modify the stiffness of the microcantilever or to increase the surface area especially in biosensing applications. As we have shown, modifying the microcantilever geometry can lead to nonintuitive results; for example, the quality factor of
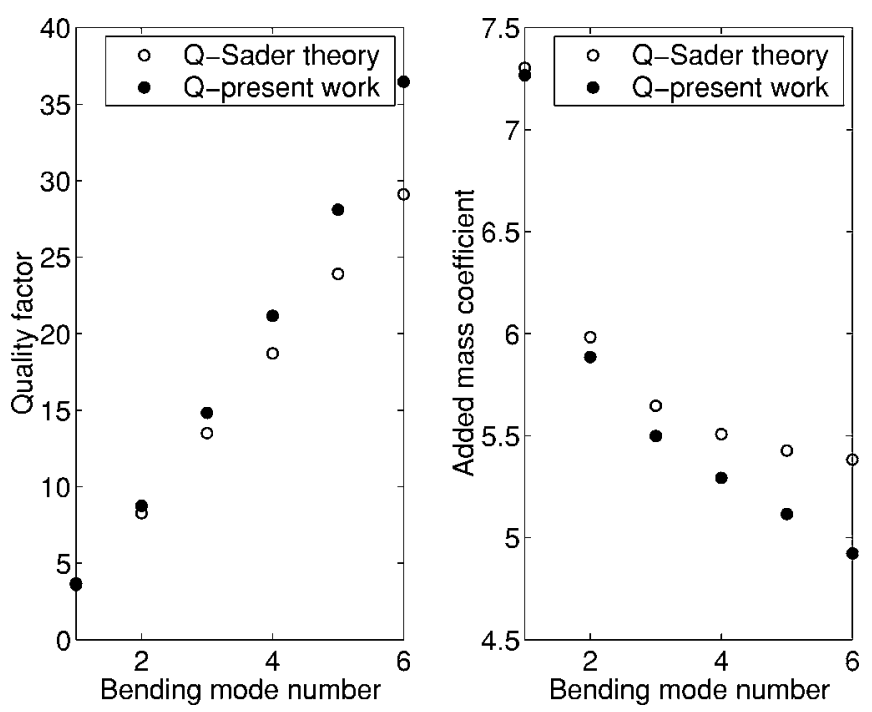

FIG. 4. (Color online) Comparison of quality factors and added mass coefficients for higher modes by Sader theory (Ref. 16) and present work.

the $\mathrm{C} 4$ microcantilever is greater than that of $\mathrm{C} 3$, even though their surface areas are identical. Based on the results in the previous sections it is clear that increasing the effective width of the microcantilever leads to increased quality factors. To maximize the effective width the use of necks or slots in microcantilevers should be minimized. As shown earlier, wider microcantilevers possess higher dry and wet natural frequencies in comparison to narrower microcantilevers. Thus, shorter and wider microcantilevers without additional edges or slots are expected to maximize both the quality factor and the wet natural frequency in viscous fluids. However, shorter and wider microcantilevers also lead to increased static stiffness of the microcantilever which may be disadvantageous for surface stress based biosensing and also for some AFM applications. Thus a compromise between the static stiffness of the microcantilever and its quality factors and wet natural frequencies would be necessary for optimal microcantilever design for operation in fluids.

\section{E. Quality factors and added mass coefficients for higher bending modes}

Having discussed the fundamental mechanisms of fluid dissipation and added mass and their dependence on microcantilever geometry, we now focus on the variation of these quantities with mode shape. The semianalytical model of Ref. ${ }^{16}$ is only applicable for the first few modes of vibration. The model developed here is fully three dimensional and can overcome the limitations of the semianalytical model. ${ }^{16} \mathrm{We}$ compare the semianalytical and computational predictions of added mass coefficients and the quality factors for higher modes of vibration up to the sixth bending mode.

A comparison of the quality factors [Eq. (5a)] and added mass coefficients [Eq. (6)] between the semianalytical model of Sader $^{16}$ and the full three-dimensional simulation in ADINA is presented in Fig. 4. As seen in the figure, the errors in the estimation of the quality factors and the added mass coefficients increase with increase in mode number. In general, the semianalytical model ${ }^{16}$ underpredicts the quality 


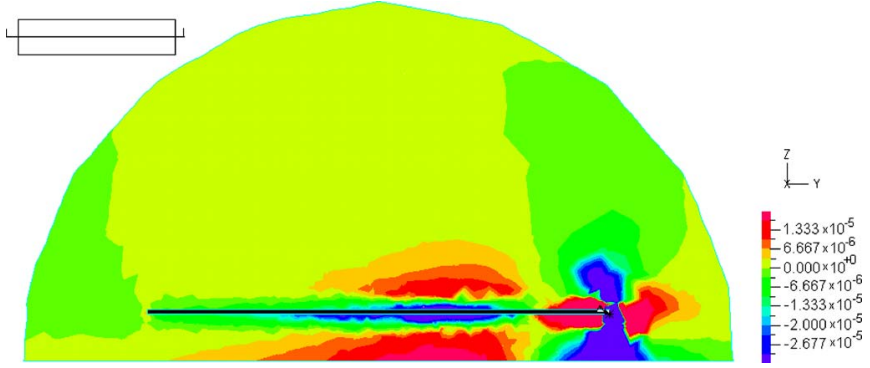

FIG. 5. (Color online) Shear stress $\left(\tau_{y z}\right)$ Pascal in the fluid in $y z$ plane for $\mathrm{C} 2$ microcantilever when the microcantilever vibrates $20 \mu \mathrm{m}$ away from the surface. This stress distribution is plotted at the instant when the microcantilever is going up in its equilibrium position after one complete cycle of oscillation.

factors and overpredicts the added mass coefficients.

The assumption of two-dimensional flow in the semianalytical model ${ }^{16,17}$ is the main source of this discrepancy. Because the semianalytical model does not permit fluid flow in the axial direction of the microcantilever, it overpredicts the pressures and pressure gradients in the fluid on the transverse cross-sectional plane of the microcantilever. Likewise, because the fluid is not allowed to leave the cross-sectional plane, more fluid is dragged with the microcantilever in the semianalytical model compared to the real three-dimensional situation. Consequently the semianalytical model overpredicts the added mass coefficients and underpredicts the wet natural frequencies.

\section{MICROCANTILEVER VIBRATING CLOSE TO A SURFACE}

In many MEMS and AFM applications microcantilevers are either fabricated (or intentionally moved) in the close vicinity of a surface. Moreover in most AFM systems the microcantilever is oriented at a small angle (typically $11^{\circ}$ ) relative to the surface being scanned. Often the nearness of the microcantilever to a surface can affect significantly the quality factors and wet frequencies of the microcantilever. In

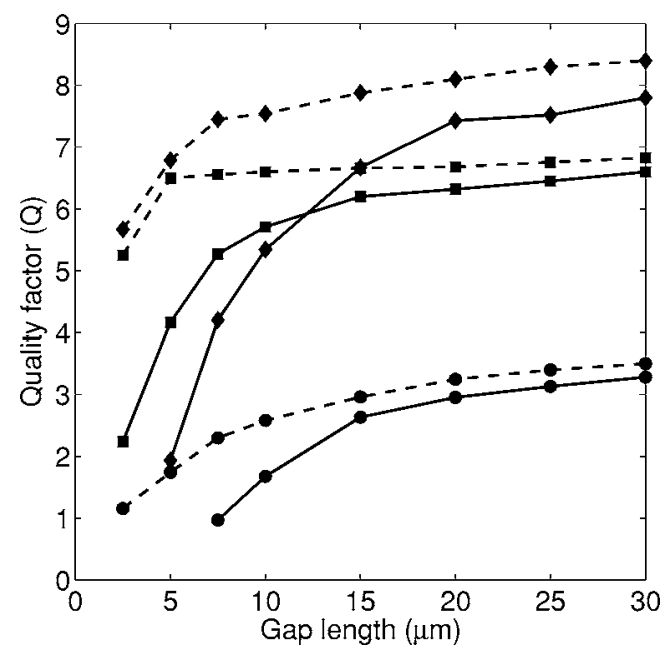

FIG. 6. (Color online) Comparison of quality factors for the first and the second bending mode and the first torsional mode for $\mathrm{C} 2$ microcantilever in water. Circles: first bending, diamonds: second bending, and squares: first torsion. Solid lines: parallel orientation, and dashed lines: $11^{\circ}$ orientation.

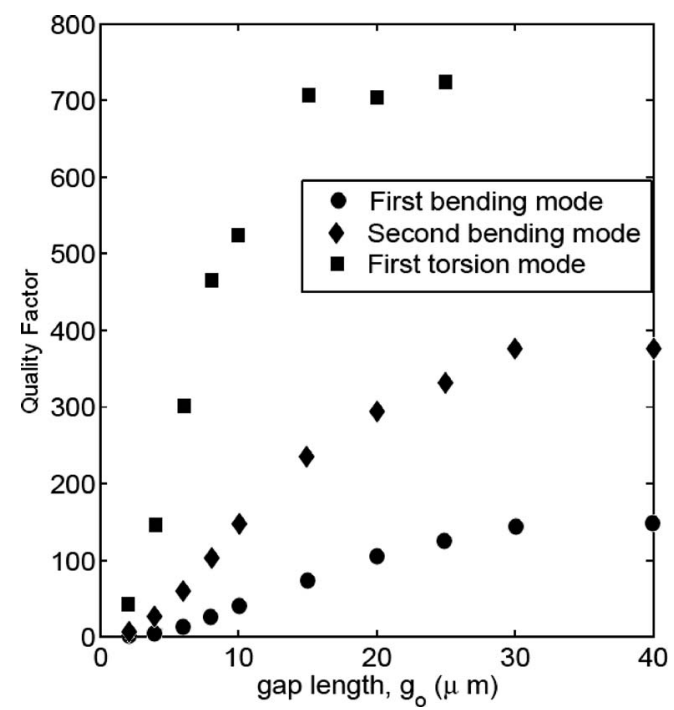

FIG. 7. (Color online) Comparison of quality factors for the first and the second bending mode and the first torsional mode for $\mathrm{C} 2$ microcantilever in air.

this section we will explore the mechanisms by which the closeness and orientation to the surface affects the hydrodynamic loading on the microcantilever.

\section{A. Quality factors and their dependence on gap width}

To understand how fluid viscous dissipation is modified when the microcantilever is brought close to a surface, we plot in Fig. 5 the fluid shear stresses in the longitudinal cross-sectional plane of the $\mathrm{C} 2$ microcantilever when it is placed $20 \mu \mathrm{m}$ from a surface [Fig. 1(b)]. The stress distribution in the fluid is plotted from the transient ADINA simulations at a time instant when the microcantilever is at its equilibrium position but swinging upwards. Comparing the stress distribution in Fig. 5 with that of Fig. 3(a) it is clear that significant fluid shear occurs in the gap between the microcantilever and the surface. As the microcantilever is brought closer, this squeeze film damping continues to increase. Because the squeeze film effects occur in addition to the localized fluid shear at the microcantilever edges, the fluid damping is expected to increase significantly upon approach toward a surface. Moreover, because it is influenced by the relative motion between the microcantilever and the surface, the squeeze film effect is expected to be mode shape dependent.

To quantify this effect the quality factors in air and water of the $\mathrm{C} 2$ microcantilever for the first and second bending

TABLE II. Convergence (mesh independence) study for C2 microcantilever $\left(197 \times 29 \times 2 \mu \mathrm{m}^{2}\right)$ made of single crystal silicon immersed in water for first bending mode.

\begin{tabular}{ccc}
\hline \hline & $\begin{array}{c}\text { Fraction on right side of Eq. (A1) } \\
\text { (accepted when } \leqslant 0.01)\end{array}$ & $Q$ \\
\hline 56000 & $\ldots$ & 2.8 \\
75000 & 0.079 & 3.5 \\
84000 & 0.034 & 3.7 \\
105000 & 0.004 & 3.7 \\
\hline \hline
\end{tabular}


modes as well as for the first torsional mode are computed and plotted as a function of gap width in Figs. 6 and 7. As described earlier all computations are performed using transient simulations in ADINA with same mesh density as in Table II and sufficiently small time steps.

Several observations can be made from Figs. 6 and 7 . The quality factors of the bending modes gradually decrease as the gap width decreases; however, for the torsional mode, the quality factor remains unaffected and reduces suddenly for gap widths less than $15 \mu \mathrm{m}$. This result can be explained as follows. When the microcantilever vibrates in a particular mode, an unsteady Stokes boundary layer is set up around the microcantilever. The boundary layer thickness is known to be proportional to $\sqrt{(\nu / \omega)},{ }^{30,31}$ where $\omega$ is the oscillation frequency and $\nu$ is the kinematic viscosity. When the gap between the microcantilever and the surface becomes comparable to the unsteady boundary layer thickness, the squeeze film effects start to emerge, thereby altering the hydrodynamic loading mechanisms. Because the torsional mode has the highest natural frequency of the three modes considered, it generates a very thin unsteady boundary layer around the microcantilever. Consequently a microcantilever oscillating in its torsional mode can approach much closer to the surface without being influenced by the squeeze film effect. This phenomenon is even more pronounced in air where the quality factor of the torsional mode remains nearly constant at $\sim 700$ for gaps greater than $15 \mu \mathrm{m}$, but plunges to $\sim 450$ when the gap reduces to $10 \mu \mathrm{m}$.

The results in Figs. 6 and 7 bear significant implications for force spectroscopy applications in AFM. For instance, changes in tip-sample dissipation are measured by bringing the cantilever close to the sample. However, some of the change in measured dissipation may be attributable to changes in hydrodynamic damping, especially while using higher cantilever modes or while approaching the sample over large distances. To avoid this, the imaging tip on the microcantilever needs to be sufficiently long so that the microcantilever is forced to stay a minimum distance away from the surface, thereby ensuring that the microcantilever quality factor is unaffected by the presence of the surface. For the C2 type microcantilever in water or air investigated in Figs. 6 and 7, the quality factors of the bending modes do not seem to change significantly for gap widths greater than $30 \mu \mathrm{m}$. Consequently a $30 \mu \mathrm{m}$ long imaging tip would ensure that the quality factors do not change due to hydrodynamic loading upon approach to the sample surface.

\section{B. Added mass coefficients and dependence on gap width}

In addition to the quality factors, the proximity to a surface also modifies the wet natural frequencies, and consequently the hydrodynamic added mass coefficients, of the different modes. This is illustrated in Fig. 8 where the ADINA computed wet (in water) and dry (in vacuum) frequencies are used to plot the added mass coefficients as a function of the gap width.

An important observation from Fig. 8 is that the added mass coefficient is greatest for the first bending mode fol-

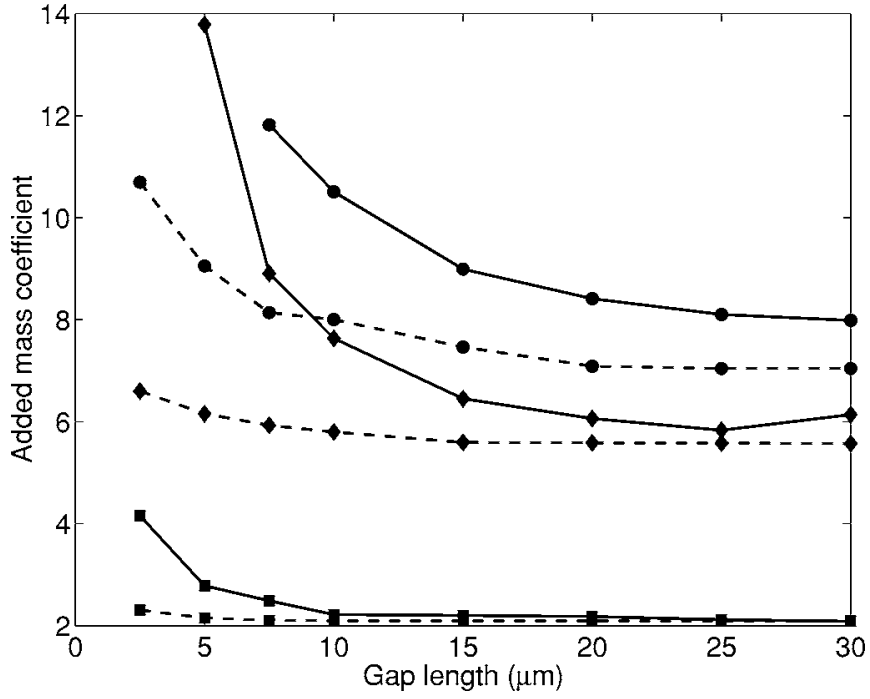

FIG. 8. (Color online) Comparison of added mass coefficients for the first and the second bending mode and the first torsional mode for $\mathrm{C} 2$ microcantilever in water. Circles: first bending, diamonds: second bending, and squares: first torsion. Solid lines: parallel orientation and dashed lines: $11^{\circ}$ orientation.

lowed in decreasing order by the second bending and torsional modes. This is a known result from the theory of hydrodynamic loading of elastic structures ${ }^{16}$ by which the lowest modes are expected to cause the largest bulk fluid motion in their vicinity, thereby increasing the added mass coefficients. In addition, the added mass coefficients increase as the gap is decreased for all the three modes. This result is also expected because the motion of the thin layer of trapped fluid between the microcantilever and the surface becomes increasingly constrained as the gap is decreased. As the gap between the microcantilever and the surface decreases, the boundary layers generated on both the cantilever and the nearby surface occupy a greater fraction of the gap. Thus with decrease in gap length, a greater fraction of the fluid in the gap is subjected to viscous drag and greater effort is needed to move this constrained fluid in the gap thereby increasing the added mass coefficient. This in turn leads to an increasing added fluid mass effect as the gap decreases.

This change in frequency upon approach to a surface also has significant implications for force spectroscopy in liquids using AFM. Usually, shifts in microcantilever resonance frequency occur over tens of nanometers due to tipsample interaction forces. ${ }^{4,32,33}$ However, there is evidence in the literature where the long range interaction forces span over several mocrometers. ${ }^{34}$ Any changes in natural frequency due to the changing added mass coefficient over several micrometers of gap may be incorrectly attributed to the presence of long range interaction forces. ${ }^{34}$ Moreover, the shift in natural frequency encountered upon approach to the surface can be mistakenly attributed to the decreased quality factors. The results in Fig. 8 clearly show that in addition to a decreased quality factor, the wet natural frequency intrinsically changes due to the presence of a surface in close proximity to the microcantilever. 


\section{Effects of microcantilever orientation}

In AFM applications, the microcantilever is usually oriented at an angle to the surface being imaged. In order to investigate the effects of orientation on the hydrodynamic loading, quality factors and added mass coefficients are plotted as shown in Figs. 6 and 8 for a microcantilever immersed in water at an angle of $\gamma=11^{\circ}$ to the surface. Clearly for the same gap between the microcantilever end and the surface, the quality factors of the inclined microcantilever are significantly greater than for the case when the microcantilever is parallel to the surface. Likewise, the added mass coefficients decrease when the microcantilever is inclined with respect to the surface. Microcantilever inclination increases the average gap between the microcantilever and the surface thereby mitigating the additional hydrodynamic loading due to the proximity of the surface. While this may suggest that further inclination of microcantilevers may be advantageous for liquid AFM, this also introduces unwanted lateral forces between the tip and the sample. Choosing an optimal microcantilever orientation for liquid AFM therefore requires a compromise between increasing quality factors and wet natural frequencies on one hand and increasing unwanted lateral forces on the other hand.

\section{CONCLUSIONS}

A fully three-dimensional finite element-based fluid structure interaction model has been developed to predict the hydrodynamic loading of microcantilevers in viscous fluids. The model predictions have been thoroughly benchmarked against several known experimental and theoretical results, and found to be in excellent agreement. It is found that the main dissipation mechanism in a viscous fluid arises from localized fluid shear near the edges of the vibrating microcantilever. When placed in close proximity to a surface, fluid dissipation arises due to a combination of squeeze film effects and localized fluid shear near the edges. Modifying the microcantilever geometry can lead to unexpected changes in the quality factors and wet natural frequencies. In general, to maximize the quality factors and wet natural frequencies, the effective width needs to be maximized, the presence of slots and edges minimized, and the length minimized. It is also shown that existing two-dimensional semianalytical models tend to underpredict the quality factors and overpredict the added mass coefficients especially for the higher modes of operation. The quality factors and wet natural frequencies of different modes decrease upon approach to a rigid surface, while the added mass coefficient increases. The variation of quality factors with gapwidth is more gradual for the bending modes than for the torsional mode, a result which is explained in terms of the unsteady viscous boundary layer surrounding the microcantilever. Finally, orienting the microcantilever at an angle to a proximal surface increases the quality factors and wet natural frequencies in comparison to the case when the microcantilever is parallel to the surface.

The use of three-dimensional fluid-structure interaction models is a powerful tool for the optimization of complicated microcantilever geometries, for the verification of the limita- tions of simple analytical models, and to understand the fundamental physics of fluid-structure interaction at the microscale.

\section{ACKNOWLEDGMENTS}

The authors acknowledge the financial support of the Cooling Technologies Research Center, a National Science Foundation Industry/University Cooperative Research Center at Purdue University. One of the authors (A.R.) also acknowledges financial support from the National Science Foundation through Grant Nos. 0134455CMS and 0409660-CMS. The authors also thank Professor Cagri Savran (School of Mechancial Engineering, Purdue University) for useful discussions regarding microcantilever design for biosensors.

\section{APPENDIX A: DOMAIN, TIME STEP, AND MESH INDEPENDENCE STUDY}

In order to verify the accuracy of the model, computations are performed for two silicon microcantilevers, referred to as calibrated microcantilevers and denoted by $\mathrm{C} 1$ $\left(397 \times 20 \times 2 \mu \mathrm{m}^{3}\right)$ and $\mathrm{C} 2\left(197 \times 20 \times 2 \mu \mathrm{m}^{3}\right)$ in Ref. 17 (Young's modulus $E_{b}=169 \mathrm{GPa}$, density $\rho_{b}=2320 \mathrm{~kg} \mathrm{~m}^{-3}$, and Poisson's ratio $\nu=0.25$ for silicon).

While results presented in this paper are from full threedimensional ADINA simulations, the domain independence and time-step independence studies are performed on the two-dimensional model [Fig. 1(a)]. This leads to a more conservative choice of the domain parameters $(r$ and $R)$ and the time step because the two-dimensional model is subjected to higher pressure and velocity gradients compared to the fully three-dimensional model. The mesh densities, domain parameters $r$ and $R$, and time steps chosen based on these twodimensional studies will be used for fully three-dimensional computations presented later. To confirm that the convergence studies performed with two-dimensional models are appropriate for three dimensions, we will demonstrate excellent agreement of the three-dimensional ADINA computations with prior experimental and analytical results.

\section{Domain independence}

Domain independence is verified by varying $r$ and $R$ [Fig. 1(a)] in the model. First the eigenvalue convergence is checked for the elastic beam in vacuum and the in vacuo mode shapes are obtained. Then the microcantilever nodes are provided an initial velocity proportional to a specific mode shape in the fluid. A maximum tip velocity of $10 \mu \mathrm{m} / \mathrm{s}$ is used in all the simulations. The parameters $r$ and $R$ are then varied separately and the transient tip deflection is recorded for every case. The metric used for checking convergence is

$$
\sqrt{\sum_{i=1}^{i=N}\left(x_{2, i}-x_{1, i}\right)^{2}} \leqslant 0.01 \sqrt{\sum_{i=1}^{i=N} x_{1, i}^{2}},
$$

where $x_{1, i}$ is the nodal displacement at the tip of the microcantilever recorded at $i=1, \ldots, N$ instants of time during the first oscillation cycle of the microcantilever transient motion, 
TABLE III. Comparison of predictions from the present work for bending modes of microcantilevers $\mathrm{C} 1$ and $\mathrm{C} 2$ in different fluids with the experimental and semianalytical results of Ref. 17. In calculating the percentage error the experimental value was taken as the base. Superscript wet refers to the values in fluid and pw stands for present work.

\begin{tabular}{|c|c|c|c|c|c|c|c|c|c|c|}
\hline Fluid & $f_{\text {expt }}^{\mathrm{wet}}$ & $f_{\text {anal }}^{\mathrm{wet}}$ & $\begin{array}{c}\text { Error }_{\text {anal }} \\
(\%)\end{array}$ & $\begin{array}{c}f_{\mathrm{pw}}^{\mathrm{wet}} \\
(\mathrm{kHz})\end{array}$ & $\begin{array}{c}\text { Error }_{\mathrm{pw}} \\
(\%)\end{array}$ & $Q_{\text {expt }}$ & $Q_{\text {anal }}$ & $\begin{array}{c}\text { Error }_{\text {anal }} \\
(\%)\end{array}$ & $Q_{\mathrm{pw}}$ & $\begin{array}{c}\text { Error }_{\mathrm{pw}} \\
(\%)\end{array}$ \\
\hline \multicolumn{11}{|c|}{ Microcantilever $\mathrm{C} 1$} \\
\hline Air Mode 1 & 17.36 & 17.53 & 0.98 & 17.32 & 0.23 & 55.5 & 55.0 & 0.90 & 55.38 & 0.22 \\
\hline Air Mode 2 & 109.3 & 109.3 & 0.00 & 109.2 & 0.10 & 170 & 169 & 0.59 & 172 & 1.16 \\
\hline Acetone & 6.35 & 6.17 & 3.0 & 6.36 & 0.16 & 2.9 & 3.0 & 3.45 & 3.14 & 8.28 \\
\hline $\mathrm{CCl}_{4}$ & 4.22 & 4.00 & 5.0 & 4.33 & 2.61 & 2.0 & 2.1 & 5.00 & 2.12 & 6.20 \\
\hline Water & 5.04 & 4.79 & 5.0 & 5.08 & 0.79 & 2.0 & 2.0 & 0.00 & 2.04 & 1.90 \\
\hline \multicolumn{11}{|c|}{ Microcantilever C2 } \\
\hline Air Mode 1 & 69.87 & 70.31 & 0.63 & 70.49 & 0.89 & 136 & 131 & 3.68 & 144.8 & 6.47 \\
\hline Air Mode 2 & 438.5 & 438.7 & 0.04 & 441.6 & 0.71 & 395 & 357 & 9.62 & 367 & 7.09 \\
\hline Acetone & 29.5 & 28.0 & 5.08 & 29.05 & 1.53 & 5.1 & 5.3 & 3.92 & 5.46 & 7.06 \\
\hline $\mathrm{CCl}_{4}$ & 20.8 & 19.4 & 6.73 & 20.64 & 0.77 & 3.7 & 3.7 & 0.00 & 3.66 & 1.08 \\
\hline Water & 25.2 & 23.5 & 6.75 & 25.0 & 0.80 & 3.7 & 3.5 & 5.41 & 3.7 & 0.00 \\
\hline
\end{tabular}

and $x_{2, i}$ is the array of nodal displacements at the tip at exactly the same instants of time when the computations are performed with a finer time step or denser mesh or larger $r$. Specifically the $N=100$ time instants (except for the time step independence study, discussed in Appendix A 2) are chosen from the first two cycles of oscillation. Convergence is checked for $r=(0.9 / \sqrt{2}) a, \quad(1 / \sqrt{2}) a, \quad(1.1 / \sqrt{2}) a$, and $(1.2 / \sqrt{2}) a$, with $R=1.3 a$, where $a$ is the length of the microcantilever. In other words, as the viscous fluid domain is expanded we expect to see a convergence in the microcantilever transient motion. For all these cases criterion (A1) is satisfied. Similarly for the $R$ independence, with $r=(1.1 / \sqrt{2}) a$, convergence is checked for $R=(1.4 / \sqrt{2}) a$, $(1.7 / \sqrt{2}) a$, and $(2 / \sqrt{2}) a$. The convergence criterion (A1) is again satisfied indicating that the fluid viscous effects are localized near the microcantilever and that it suffices to terminate the viscous fluid domain at the finite radius chosen.

\section{Time step independence}

The time step $d t$ required to resolve sufficiently the transient structural and fluid motions is suggested in Ref. 25 to be $T / 20$, where $T$ is the time period of oscillation. The time step independence is checked for time steps $d t=T / 25, T / 40$, and $T / 50$. The convergence criterion (A1) is satisfied for all cases indicating that $d t=T / 25$ is a sufficiently small time step to track the microcantilever transient motion accurately.

\section{Mesh independence}

The mesh independence study is performed for the full three-dimensional model. The fluid mesh independence is checked for convergence by increasing the number of elements in the fluid domain and thereby achieving finer mesh density. For this case, $r=0.9 \sqrt{3 / 2} a$ and $R=1.5 \sqrt{3 / 2} a$ are chosen and the results obtained are listed in Table II. The quality factor $Q$ (the method used for determining the quality factor from the transient simulations is discussed in detail in the next section) values of the first bending mode are also computed for the corresponding cases as an additional check of convergence.

\section{Computational procedure}

The microcantilever is prescribed initial velocities proportional to one of its mode shapes. The small amplitude of vibration (due to small initial velocities) also ensures that the quadratic nonlinearities in the Navier-Stokes equations are not excited and initial excitation of the microcantilever decays as a linear damped oscillator. This allows the use of a linear damping model. The transient tip displacement is then used to determine the natural frequency and quality factor of the particular vibration mode. This procedure enables efficient computation of the damping and natural frequencies even for higher modes with low quality factors.

The transient tip displacement is fitted in MATLAB (Ref. 35 ) with the damped transient response of a linear damped oscillator,

$$
x(t)=X e^{-\omega_{n} t / 2 Q_{n}} \sin \left(\omega_{n} t \sqrt{1-1 / 4 Q_{n}^{2}}\right),
$$

where $X, \omega_{n}$, and $Q_{n}$ are, respectively, the amplitude of vibration, undamped natural frequency, and the quality factor. A nonlinear least-squares fit is computed with a robust bisquare regression scheme using the Gauss-Newton algorithm. This yields the natural frequency $\omega_{n}$ and quality factor $Q_{n}$ of a particular mode of vibration.

\section{APPENDIX B: VALIDATION OF THE COMPUTATIONAL MODEL}

In this section the numerical model is validated against published experimental $^{17}$ and analytical ${ }^{17,18,26}$ results for the case of rectangular microcantilevers for different modes of vibration in different fluids.

TABLE IV. Properties of fluids used in Ref. 17 and the present ADINA computations.

\begin{tabular}{lcc}
\hline \hline \multicolumn{1}{r}{ Fluid } & Dynamic viscosity $\left(\mathrm{kg} \mathrm{m}^{-1} \mathrm{~s}^{-1}\right)$ & Density $\left(\mathrm{kg} \mathrm{m}^{-3}\right)$ \\
\hline Air & $1.86 \times 10^{-5}$ & 1.18 \\
Acetone & $3.08 \times 10^{-4}$ & 785 \\
$\mathrm{CCl}_{4}$ & $8.79 \times 10^{-4}$ & 1590 \\
Water & $8.59 \times 10^{-4}$ & 997 \\
\hline
\end{tabular}


TABLE V. Comparison of predictions from the present work of the first torsional mode of different microcantilevers in air with the experimental results of Ref. 26 and semianalytical results of Ref. 18. pw stands for present work.

\begin{tabular}{|c|c|c|c|c|c|c|}
\hline Microcantilevers & $f_{\text {expt }}^{\text {wet }}$ & $f_{\mathrm{pw}}^{\mathrm{wet}}$ & Error $(\%)$ & $Q_{\text {anal }}$ & $Q_{\mathrm{pw}}$ & $\begin{array}{c}\text { Error }_{\mathrm{pw}} \\
(\%)\end{array}$ \\
\hline \multicolumn{7}{|c|}{ Comparison with experimental results of Ref. 26} \\
\hline $\mathrm{R} 1$ & 393.4 & 390.8 & 0.66 & 104.0 & 110.1 & 5.87 \\
\hline $\mathrm{R} 2$ & 258.3 & 256.9 & 0.54 & 167 & 151.1 & 9.52 \\
\hline \multicolumn{7}{|c|}{ Comparison with semianalytical results of Ref. 18} \\
\hline Microcantilevers & $\Pi$ & $\operatorname{Re}$ & $Q_{\text {anal }}$ & $Q_{\mathrm{pw}}$ & $\begin{array}{c}\text { Error }_{\mathrm{pw}} \\
(\%)\end{array}$ & \\
\hline H1 & 0.02 & 10 & 60 & 61.14 & 1.9 & \\
\hline $\mathrm{H} 2$ & 0.005 & 10 & 237 & 251.1 & 5.9 & \\
\hline
\end{tabular}

First consider the predictions for the hydrodynamic loading on the bending modes of microcantilevers. Experimental results for $\mathrm{C} 1$ and $\mathrm{C} 2$ from Ref. 17 in air, water, acetone, and carbon tetrachloride are compared in Table III with the present computational predictions. Properties of the fluids used are listed in Table IV. The predictions from the present work are within $2 \%$ of the experimental frequencies and within $10 \%$ of the quality factors for the lowest two bending modes.

Experimental data for the torsional modes in air are available from Ref. 26, but for microcantilevers with imaging tips. Two such microcantilevers, R1 $\left(197 \times 19 \times 0.64 \mu \mathrm{m}^{3}\right)$ and $\mathrm{R} 2\left(422 \times 29 \times 1.15 \mu \mathrm{m}^{3}\right)$, are modeled in ADINA without the tips. Because the thicknesses for these two microcantilevers are not reported in Ref. 26, the thicknesses were adjusted so that the torsional frequencies are within $1 \%$ of the experimental frequencies. The comparison of torsional quality factors for the first mode for $\mathrm{R} 1$ and $\mathrm{R} 2$ is provided in Table $\mathrm{V}$. The quality factors are once again within $10 \%$ of the experimental quality factors. In order to compare the current predictions for torsional mode quality factors with semianalytical results in the prior literature, ${ }^{18}$ we turn our attention to two hypothetical microcantilevers $\mathrm{H} 1 \quad\left(344 \times 15.73 \times 0.4 \mu \mathrm{m}^{3}\right)$ and $\mathrm{H} 2$ $\left(131 \times 4.92 \times 0.5 \mu^{3}\right)$ that possess the same nondimensional terms as Fig. 4 of Ref. 18. The microcantilevers made of silicon crystal are assumed to be vibrated in air. Table V presents the comparison of quality factors of these two hypothetical microcantilevers. The comparisons are within $6 \%$ of the semianalytical model of Ref. 26 for the two microcantilevers considered.

${ }^{1}$ C. A. J. Putman, K. O. Van der Werf, B. G. De Grooth, N. F. Van Hulst, and J. Greve, Appl. Phys. Lett. 64, 2454 (1994).

${ }^{2}$ N. J. Tao, S. M. Lindsay, and S. Lees, Biophys. J. 63, 1165 (1992).

${ }^{3}$ P. K. Hansma et al., Appl. Phys. Lett. 64, 1738 (1994).

${ }^{4}$ M. J. Higgins, C. K. Riener, T. Uchihashi, J. E. Sader, R. McKendry, and S. P. Jarvis, Nanotechnology 16, 85 (2005).

${ }^{5}$ J. Tamayo, A. D. L. Humphris, and M. J. Miles, Appl. Phys. Lett. 77, 582 (2000).

${ }^{6}$ F. M. Battiston, J. P. Ramseyer, H. P. Lang, M. Baller, C. Gerber, J. K. Gimzewski, E. Meyer, and H. J. Guntherodt, Sens. Actuators B 77, 122
(2001).

${ }^{7}$ H. P. Lang, M. Hegner, E. Meyer, and C. Gerber, Nanotechnology 13, 29 (2002).

${ }^{8}$ C. A. Savran, T. P. Burg, J. Fritz, and S. R. Manalis, Appl. Phys. Lett. 83, 1659 (2003).

${ }^{9}$ K. L. Ekinci, Y. T. Yang, and M. L. Roukes, J. Appl. Phys. 95, 2682 (2004).

${ }^{10}$ K. L. Ekinci, X. M. H. Huang, and M. Roukes, Appl. Phys. Lett. 84, 4469 (2004).

${ }^{11}$ K. M. Hansen, H. F. Ji, G. Wu, R. Datar, R. Cote, A. Majumdar, and T. Thundat, Anal. Chem. 73, 1567 (2001).

${ }^{12}$ A. H. Nayfeh and M. I. Younis, J. Micromech. Microeng. 14, 170 (2004).

${ }^{13}$ H. Hosaka, K. Itao, and S. Kuroda, Evaluation of Energy Dissipation Mechanisms in Vibrational Microactuators, Proceedings of the IEEE Micro Electro Mechanical Systems (Elsevier, Japan, 1994), p. 193-198.

${ }^{14}$ H. Hosaka, K. Itao, and S. Kuroda, Sens. Actuators, A 49, 87 (1995).

${ }^{15}$ W. H. Chu, Vibration of Fully Submerged Cantilever Plates in Water, South-West Research Institute, Technical Report No. 2, 1963.

${ }^{16}$ J. E. Sader, J. Appl. Phys. 84, 64 (1998).

${ }^{17}$ J. W. M. Chon, P. Mulvaney, and J. E. Sader, J. Appl. Phys. 87, 3978 (2000).

${ }^{18}$ C. P. Green and J. E. Sader, J. Appl. Phys. 92, 6262 (2002).

${ }^{19}$ T. Terasawa, Y. Kawamura, K. Sato, and S. Tanaka, Bull. Jpn. Soc. Precis. Eng. 22, 49 (1988).

${ }^{20}$ K. Kokubun, H. Murakami, Y. Toda, and M. Ono, Vacuum 34, 731 (1984).

${ }^{21}$ F. R. Blom, S. Bouwstra, M. Elwenspoek, and J. H. J. Fluitman, J. Vac. Sci. Technol. B 10, 19 (1992).

${ }^{22}$ A. Maali, C. Hurth, R. Boisgard, C. Jai, T. Cohen-Bouhacina, and J. P. Aime, J. Appl. Phys. 97, 074907 (2005).

${ }^{23}$ C. Green and J. Sader, Phys. Fluids 17, 73102 (2005).

${ }^{24}$ E. O. Tuck, J. Eng. Math. 3, 29 (1969).

${ }^{25}$ ADINA System Online Manuals, ADINA R\&D, 2003.

${ }^{26}$ C. P. Green, H. Lioe, J. P. Cleveland, R. Proksch, P. Mulvaney, and J. E. Sader, Rev. Sci. Instrum. 75, 1988 (2004).

${ }^{27}$ The Reynolds number is traditionally defined as the ratio of the nonlinear convective term to the viscous term in Navier-Stokes equation.

${ }^{28}$ L. Rosenhead, Laminar Boundary Layers (Dover, New York, 1963).

${ }^{29}$ J. E. Sader, I. Larson, P. Mulvaney, and L. R. White, Rev. Sci. Instrum. 66, 3789 (1995).

${ }^{30}$ G. K. Bachelor, Fluid Dynamics (Cambridge University Press, Cambridge, UK, 1974).

${ }^{31} \mathrm{H}$. Schlichting and K. Gersten, Boundary-Layer Theory, 8th ed. (Springer, Berlin, 2001).

${ }^{32}$ J. E. Sader and S. P. Jarvis, Appl. Phys. Lett. 84, 1801 (2004).

${ }^{33}$ J. E. Sader, T. Uchihashi, M. J. Higgins, A. Farrell, Y. Nakayama, and S. P. Jarvis, Nanotechnology 16, 94 (2005).

${ }^{34}$ J. Colchero, A. Gil, and A. Baro, Phys. Rev. B 64, 245403 (2001).

${ }^{35}$ Matlab Online Documentation Set, The MathWorks, (2005). 